\title{
Improved efficiency of Kiltz07-KEM
}

\author{
Xianhui $\mathrm{Lu}^{1}$, Xuejia $\mathrm{Lai}^{2}$, Dake $\mathrm{He}^{1}$ \\ Email:luxianhui@gmail.com \\ 1:School of Information Science \& Technology, SWJTU, Chengdu, China \\ 2:Dept. of Computer Science and Engineering, SJTU, Shanghai, China
}

\begin{abstract}
Kiltz proposed a practical key encapsulation mechanism(Kiltz07-KEM) which is secure against adaptive chosen ciphertext attacks(IND-CCA2) under the gap hashed Diffie-Hellman(GHDH) assumption[8]. We show a variant of Kiltz07-KEM which is more efficient than Kiltz07-KEM in encryption. The new scheme can be proved to be IND-CCA2 secure under the same assumption, GHDH.
\end{abstract}

Keywords: KEM, IND-CCA2, GHDH

\section{Introduction}

Security against adaptive chosen ciphertext attacks (IND-CCA2 security) [1-3] is now commonly accepted as the standard security notion for public key encryption schemes. Currently, most of the practical IND-CCA2 secure public key encryption schemes in standard model are variants of ElGamal[4] scheme. Cramer and Shoup[5,6] proposed the first provably IND-CCA2 secure practical public key encryption scheme based on the decisional Diffie-Hellman(DDH) assumption in the standard model. This was further improved by Kurosawa and Desmedt and yield a more efficient scheme(KD04)[7]. Kiltz proposed a IND-CCA2 secure KEM(key encapsulation mechanism) under the Gap Hashed Diffie-Hellman(GHDH) assumption[8]. Combined with a redundancy-free DEM(data encapsulation mechanism) it will yield a IND-CCA2 secure hybrid encryption scheme more efficient than KD04.

\subsection{Our Contributions}

We show a variant of Kiltz07-KEM which can be proved to be IND-CCA2 secure under the same assumption, GHDH. The new scheme is similar to Kiltz07-KEM, while the only difference is that the second item of the ciphertext $u^{r t} v^{r}$ is replaced with $u^{r} v^{r t}$. Thus, the encryption of the new scheme only need three exponentiations. Compared with Kiltz07-KEM, the efficiency of the encryption is improved by $14.3 \%$.

\section{Definitions}

In this section we describe the definitions of KEM, GHDH assumption and target collision resistant hash function. In describing probabilistic processes, we write $x \stackrel{R}{\leftarrow} X$ to denote the action of assigning to the variable $x$ a value sampled according to the distribution $\mathrm{X}$. If $S$ is a finite set, we simply write $s \stackrel{R}{\leftarrow} S$ to denote assignment to $s$ of an element sampled from uniform distribution on $S$. If $A$ is a probabilistic algorithm and $x$ an input, then $A(x)$ denotes the output distribution of $A$ on input $x$. Thus, we write $y \stackrel{R}{\leftarrow} A(x)$ to denote of running algorithm $A$ on input $x$ and assigning the output to the variable $y$. 


\subsection{Key Encapsulation Mechanism}

A key encapsulation mechanism consists the following algorithms:

- KEM.KeyGen $\left(1^{k}\right)$ : A probabilistic polynomial-time key generation algorithm takes as input a security parameter $\left(1^{k}\right)$ and outputs a public key PK and secret key SK. We write $(\mathrm{PK}, \mathrm{SK}) \leftarrow$ $\operatorname{KEM} \cdot \operatorname{KeyGen}\left(1^{k}\right)$

- KEM.Encrypt(PK): A probabilistic polynomial-time encryption algorithm takes as input the public key PK, and outputs a pair $(K, \psi)$, where $K \in K_{D}\left(K_{D}\right.$ is the key space) is a key and $\psi$ is a ciphertext. We write $(K, \psi) \leftarrow$ KEM.Encrypt $(\mathrm{PK})$

- KEM.Decrypt(SK, $\psi$ ): A decryption algorithm takes as input a ciphertext $\psi$ and the secret key SK. It returns a key $K$. We write $K \leftarrow$ KEM.Decrypt(SK, $\psi$ ).

We require that for all (PK,SK) output by $\operatorname{KEM} \cdot \operatorname{KeyGen}\left(1^{k}\right)$, all $(K, \psi) \in[\operatorname{KEM}$.Encrypt(PK)], we have KEM.Decrypt $(\mathrm{SK}, \psi)=K$.

A KEM scheme is secure against adaptive chosen ciphertext attacks if the advantage of any adversary in the following game is negligible in the security parameter $\mathrm{k}$ :

1. The adversary queries a key generation oracle. The key generation oracle computes $(\mathrm{PK}, \mathrm{SK}) \leftarrow$ KEM.KeyGen $\left(1^{k}\right)$ and responds with PK.

2. The adversary makes a sequence of calls to the decryption oracle. For each decryption oracle query the adversary submits a ciphertext $\psi$, and the decryption oracle responds with KEM.Decrypt(SK, $\psi)$.

3. The adversary queries an encryption oracle. The encryption oracle computes:

$$
b \stackrel{R}{\leftarrow}\{0,1\} ;\left(K_{0}, \psi^{*}\right) \leftarrow \text { PKE.Encrypt }(\mathrm{PK}) ; K_{1} \stackrel{R}{\leftarrow} K_{D} ;
$$

and responds with $\left(K_{b}, \psi^{*}\right)$.

4. The adversary continues to make calls to the decryption oracle except that it may not request the decryption of $\psi^{*}$.

5. Finally, the adversary outputs a guess $b^{\prime}$.

The adversary's advantage in the above game is $\operatorname{AdvCCA}_{\mathrm{KEM}, A}(k)=\left|\operatorname{Pr}\left[b=b^{\prime}\right]-1 / 2\right|$. If a KEM is secure against adpative chosen ciphertext attack defined in the above game we say it is IND-CCA secure.

\subsection{Gap Hashed Diffie-Hellman Assumption}

Now we review the definition of gap hashed Diffie-Hellman assumption[8]. Let $G$ be a group of large prime order $q, H: G \rightarrow\{0,1\}^{l}$ be a cryptographic hash function and consider the following two experiment:

experiments $\operatorname{Exp}_{G, H, A}^{g h d h}(l)$ :

$$
\begin{gathered}
x, y \stackrel{R}{\leftarrow} Z_{q}^{*} ; W_{1} \leftarrow\{0,1\}^{l} ; W_{0} \leftarrow H\left(g^{x y}\right) ; b \stackrel{R}{\leftarrow}\{0,1\} \\
b^{\prime} \leftarrow A^{\mathcal{O}_{d d h}()}\left(g^{x}, g^{y}, W_{b}\right) ; \text { If } b=b^{\prime} \text { return } 1 \text { else return } 0 ;
\end{gathered}
$$


Here the oracle $\mathcal{O}_{d d h}\left(g, g^{a}, g^{b}, g^{c}\right)$ returns 1 if $a b=c$ otherwise return 0; We define the advantage of the $A$ in violating the gap hashed Diffie-Hellman assumption as

$$
A d v_{G, H, A}^{g h d h}(l)=\left|\operatorname{Pr}\left[\operatorname{Exp}_{G, H, A}^{g h d h}(l)=1\right]-1 / 2\right|
$$

We say that the GHDH assumption holds if $A d v_{G, H, A}^{g h d h}(l)$ is negligible for all polynomial-time adversaries $A$.

\subsection{Target collision resistant hash function}

A $(t, \epsilon)$ target collision resistant hash function (TCR) family is a collection $\mathcal{F}$ of functions $f_{K}$ : $\{0,1\}^{n} \rightarrow\{0,1\}^{m}$ indexed by a key $K \in \mathcal{K}$ (where $\mathcal{K}$ denotes the key space), and such that any attack algorithm $A$ running in time $t$ has success probability at most $\epsilon$ in the following game:

- Key Sampling: A uniformly random key $K \in \mathcal{K}$ is chosen (but not yet revealed to $A$ ).

- A Commits: $A$ runs (with no input) and outputs a hash function input $s_{1} \in\{0,1\}^{n}$.

- Key Revealed: The key $\mathrm{K}$ is given to $A$.

- A Collides: $A$ continues running and outputs a second hash function input $s_{2} \in\{0,1\}^{n}$.

We say that $A$ succeeds in the above game if it finds a valid collision for $f_{K}$, i.e. if $s_{1} \neq s_{2}$ but $f_{K}\left(s_{1}\right)=f_{K}\left(s_{2}\right)$. We define the advantage of $A$ as $A d v T C R=\left|\operatorname{Pr}\left[f_{K}\left(s_{1}\right)=f_{K}\left(s_{2}\right): s_{1} \neq s_{2}\right]-1 / 2\right|$. We say $H$ is target collision resistant hash function if $A d v T C R$ is negligible.

\section{Variant of Kiltz07-KEM}

In this section we describe the new scheme as follow:

- KeyGen: Assume that $G$ is group of order $q$ where $q$ is a large prime number.

$$
g \stackrel{R}{\leftarrow} G ; x, y \stackrel{R}{\leftarrow} Z_{q}^{*} ; u \leftarrow g^{x} ; v \leftarrow g^{y} ; P K=(g, u, v, H, T C R) ; S K=(x, y)
$$

Where $H: G \rightarrow\{0,1\}^{l}$ is the hash function used in the GHDH assumption, $l$ is the length of the key, TCR is a target collision resistant hash function.

- Encrypt: Given PK, the encryption algorithm runs as follow:

$$
r \stackrel{R}{\leftarrow} Z_{q}^{*} ; c_{1} \leftarrow g^{r} ; t \leftarrow T C R\left(c_{1}\right) ; c_{2} \leftarrow u^{r} v^{r t} ; k \leftarrow H\left(u^{r}\right) ; \psi \leftarrow\left(c_{1}, c_{2}\right)
$$

- Decrypt: Given a ciphertext $\psi=\left(c_{1}, c_{2}\right)$ and $S K$, the decryption algorithm runs as follow:

$$
t \leftarrow T C R\left(c_{1}\right) ; \text { if }\left(c_{2}=c_{1}^{x+y t}\right) k \leftarrow H\left(c_{1}^{x}\right) \text {; else return } \perp
$$

Now we prove that the KEM above is secure against adaptive chosen ciphertext attacks:

Theorem 1. The key encapsulation above is secure against adaptive chosen ciphertext attack assuming that: (1)GHDH problem is hard in the group G, (2)TCR is a target collision resistant hash function. 
To prove the theorem, we will assume that there is an adversary $A$ that can break the hybrid encryption scheme above, TCR is a target collision resistant hash function and show how to use this adversary to construct an adversary $B$ to break the GHDH problem.

Given $\left(g, u, g^{r}, W\right), B$ runs the following key generation algorithm:

$$
y \stackrel{R}{\leftarrow} Z_{q}^{*} ; t \leftarrow T C R\left(g^{r}\right) ; v \leftarrow g^{y} u^{-1 / t}
$$

The public key that $A$ sees is $(g, u, v, T C R, H), H: G \rightarrow\{0,1\}^{l}$ is the hash function used in the GHDH assumption, $l$ is the length of the key, TCR is a target collision resistant hash function. $B$ knows $y$.

First we describe the simulation of the encryption oracle. In step 3 , B sends $\left(c_{1}=g^{r}, c_{2}=\right.$ $\left.c_{1}^{y t}, k=W\right)$ to $A$. Since $c_{2}=c_{1}^{y t}=g^{y r t}=u^{r}\left(g^{y} u^{-1 / t}\right)^{r t}=u^{r} v^{r t}$, we have that the simulation of the encryption oracle is perfect.

We now describe the simulation of the decryption oracle. Given $\left(c_{1 i}, c_{2 i}\right), B$ works as follow:

$$
t_{i} \leftarrow T C R\left(u_{1 i}\right) ; \text { if } \mathcal{O}_{d} d h\left(g, u v^{t_{i}}, c_{1 i}, c_{2 i}\right)=1 k \leftarrow H\left(\left(c_{2 i} /\left(c_{1 i}^{y t_{i}}\right)\right)^{t /\left(t-t_{i}\right)}\right) \text {; else return } \perp
$$

Let $c_{1 i}=g^{r_{i}}$, if $\mathcal{O}_{d} d h\left(g, u v^{t_{i}}, c_{1 i}, c_{2 i}\right)=1$ we have that $c_{2 i}=u^{r_{i}} v^{r_{i}} t_{i}$. Consider $k$ :

$$
\begin{gathered}
k=H\left(\left(c_{2 i} /\left(c_{1 i}^{y t_{i}}\right)\right)^{t /\left(t-t_{i}\right)}\right)=H\left(\left(u^{r_{i}} v^{r_{i} t_{i}} /\left(g^{r_{i} y t_{i}}\right)\right)^{t /\left(t-t_{i}\right)}\right) \\
=H\left(\left(u^{r_{i}}\left(g^{y} u^{-1 / t}\right)^{r_{i} t_{i}} /\left(g^{r_{i} y t_{i}}\right)\right)^{t /\left(t-t_{i}\right)}\right)=H\left(\left(u^{r_{i}\left(\left(t-t_{i}\right) / t\right)}\right)^{t /\left(t-t_{i}\right)}\right)=H\left(u^{r_{i}}\right)
\end{gathered}
$$

It is clear that the simulation of the decryption oracle is perfect. Finally, when $A$ return $b^{\prime}, B$ also output $b^{\prime}$. Let $u=g^{x}$, if $b^{\prime}=0$ it means that $k=W=H\left(g^{x r}\right)$. So, if $A$ breaks the scheme successfully, then $B$ breaks the GHDH problem successfully. That's complete the proof of theorem 1.

\section{Efficiency Analysis}

The efficiency of the new scheme and Kiltz07-KEM is listed in table 1.

Table 1. Efficiency comparison

\begin{tabular}{|c|c|c|c|c|}
\hline schemes & Encryption(exp) & Decryption(exp) & Cipher-text overhead(bit) & Assumption \\
\hline Kiltz07-KEM & $3.5(2 \exp +1$ mexp $)$ & $1.5(0 \exp +1$ mexp $)$ & $2|q|$ & GHDH \\
\hline NEW & $3(3 \exp +0$ mexp $)$ & $1.5(0 \exp +1$ mexp $)$ & $2|q|$ & GHDH \\
\hline
\end{tabular}

When tabulating computational efficiency hash function is ignored, multi-exponentiation ( mexp) is counted as 1.5 exponentiations (exp). Ciphertext overhead represents the difference between the ciphertext length and the message length, and $|q|$ is the length of a group element. It is clear that the encryption of the new scheme is about $14.3 \%$ faster than that of Kiltz07-KEM. 


\section{Conclusion}

We showed a variant of Kiltz07-KEM. The new scheme is similar to Kiltz07-KEM, while the only difference is that the second item of the ciphertext $u^{r t} v^{r}$ is replaced with $u^{r} v^{r t}$. Thus, the efficiency of the encryption is improved by $14.3 \%$.

\section{References}

1. C. Rackoff and D. Simon, Non-Interactive Zero-Knowledge Proof of Knowledge and Chosen Ciphertext Attack, Adv. in Cryptology - Crypto 1991, LNCS vol. 576, Springer- Vrlag , pp. 433-444, 1991;

2. M. Bellare, A. Desai, D. Pointcheval, and P. Rogaway, Relations Among Notions of Security for Public-Key Encryption Schemes, Adv. in Cryptology - Crypto 1998, LNCS vol. 1462, Springer-Verlag, pp. 26-45, 1998;

3. D. Dolev, C. Dwork, and M. Naor, Non-Malleable Cryptography, SIAM J. Computing, 30(2): 391-437, 2000;

4. T. ElGamal. A public key cryptosystem and signature scheme based on discrete logarithms. IEEE Transactions on Information Theory, 31:469C472, 1985.

5. R. Cramer and V. Shoup, A Practical Public Key Cryptosystem Provably Secure Against Chosen Ciphertext Attack, Adv. in Cryptology - Crypto 1998, LNCS vol. 1462, Springer- Verlag , pp. 13-25, 1998;

6. R. Cramer and V. Shoup. Design and analysis of practical public-key encryption schemes secure against adaptive chosen ciphertext attack. SIAM Journal on Computing, 33(1):167-226, 2003.

7. K. Kurosawa and Y. Desmedt, A New Paradigm of Hybrid Encryption Scheme, Adv. in Cryptology - Crypto 2004, LNCS vol. 3152, Springer-Verlag, pp. 426-442, 2004;

8. Eike Kiltz. Chosen-Ciphertext Secure Key Encapsulation based on Hashed Gap Decisional Diffie-Hellman. Proceedings of the 10th International Workshop on Practice and Theory in Public Key Cryptography, PKC 2007, pp. 282-297 LNCS 4450 (2007). Springer-Verlag. Full version available on Cryptology ePrint Archive: Report 2007/036 